\title{
Aggregate Q Wave Amplitude
}

National Cancer Institute

\section{Source}

National Cancer Institute. Aggregate Q Wave Amplitude. NCI Thesaurus. Code C117789.

An aggregate $\mathrm{Q}$ wave amplitude value based on the measurement of Q wave amplitudes from multiple beats within a sing le ECG. The method of aggregation, which can vary, is typically a measure of central tendency such as the mean. (CDISC) 